\title{
An application of the MEFM to the modified Boussinesq equation
}

\author{
Tolga Akturk \\ Department of Mathematics and Science Education, Faculty of Education, Ordu University, Turkey \\ tolgaakturkk@gmail.com
}

\section{ARTICLE INFO}

Article history:

Received: 2 June 2018

Accepted: 4 October 2018

Available Online: 15 October 2018

Keywords:

Modified Expansion Function method

Modified Boussinesq equation

The solitary wave solution

AMS Classification 2010:

$35 \mathrm{C} 07,35 \mathrm{C} 08,35 \mathrm{~J} 60$

\begin{abstract}
In this paper, some travelling wave solutions of the Modified Boussinesq (MBQ) equation are obtained by using the modified expansion function method (MEFM). When the obtained solutions are commented, trigonometric functions including hyperbolic features are obtained. The 2D and 3D graphics of the solutions have been investigated by selecting appropriate parameters. All the obtained solutions provide the MBQ equation. In this work, all mathematical calculations are done with Wolfram Mathematica software.
\end{abstract}

\section{Introduction}

The solution of nonlinear partial differential equations has a measure in real life. For this reason, many methods have been developed and applied to solve these equations. Some of these, respectively the trial equation method [1], the new function methods [2-6], the extended trial equation method [7], Kudryashov method [8], the sine-Gordon expansion method [9-10] and so on. In this study, we apply the modified expansion function method (MEFM) [11-13] to solve a nonlinear MBQ equation and find new interactions among travelling wave solutions. Boussinesq-type equations of higher order in dispersion as well as in nonlinearity are reproduced for wave-current interaction over an unbalanced bottom. There are various methods in the literature to obtain the solution of the equation. Some of those; tanh method, the modified decomposition method and bilinearization method

In Section 2, Information about the modified expansion function method will be given.

In Section 3 the modified expansion function method is applied to the MBQ equation and the new exact wave solution to this problem is obtained. The 2D and 3D graphics of the solutions were drawed by using the Mathematica software program.

The modified Boussinesq equation can be defined as follows [14-16],

$$
u_{t t}-u_{x x t t}-u_{x x}+\frac{a}{2}\left(u^{2}\right)_{x x}=0 .
$$

\section{Modified Expansion Function method}

In this part, we will be given information about MEFM. Consider the following nonlinear partial differential equation (NPDE):

$P\left(u, u^{2}, u_{x}, u_{t}, u_{x x}, u_{t t},\left(u^{2}\right)_{x x}, u_{x x t}, u_{x x t t}\right)=0$,

where $u=u(x, t)$ is unknown function, $P$ is a polynomial in $u(x, t)$ and its derivatives.

The general form of the nonlinear partial differential equation (2) is given above. By applying wave conversion to NPDE expression (3), the general form of the following nonlinear ordinary differential equation (4) is obtained.

Step 1: Consider the following travelling wave transformation:

$$
u(x, t)=u(\xi), \quad \xi=v(x-c t) .
$$

Substituting Eq. (3) into Eq. (2), gives the following nonlinear ordinary differential equation (NODE);

$N\left(u, u^{2}, \frac{d u}{d \xi}, \frac{d^{2} u}{d \xi^{2}}, \ldots\right)=0$.

Step 2: We assume the following solution;

\footnotetext{
*Corresponding author
} 
$u(\xi)=\frac{\sum_{i=0}^{m} A_{i}\left[e^{-\vartheta(\xi)}\right]^{i}}{\sum_{j=0}^{n} B_{j}\left[e^{-\vartheta(\xi)}\right]^{j}}=$

$=\frac{A_{0}+A_{1} e^{-\vartheta}+\ldots+A_{m} e^{-m \vartheta}}{B_{0}+B_{1} e^{-\vartheta}+\ldots+B_{n} e^{-n \vartheta}}$,

where $A_{i}, B_{j},(0 \leq i \leq m, 0 \leq j \leq n)$.

$m, n$ are positive integers that can be obtained by using the balancing principle.

$\vartheta^{\prime}(\eta)=e^{-\vartheta(\eta)}+k e^{\vartheta(\eta)}+\lambda$.

Eq.(6) has the following families of solutions [17]:

Family 1: When, $k \neq 0, \lambda^{2}-4 k>0$,

$\vartheta(\eta)=\ln \left(\frac{-\sqrt{\lambda^{2}-4 k}}{2 k}\right.$

$\left.\tanh \left(\frac{\sqrt{\lambda^{2}-4 k}}{2}(\eta+E E)\right)-\frac{\lambda}{2 k}\right)$.

Family 2: When, $k \neq 0, \lambda^{2}-4 k<0$,

$$
\begin{aligned}
& \vartheta(\eta)=\ln \left(\frac{\sqrt{-\lambda^{2}+4 k}}{2 k}\right. \\
& \left.\tan \left(\frac{\sqrt{-\lambda^{2}+4 k}}{2}(\eta+E E)\right)-\frac{\lambda}{2 k}\right) .
\end{aligned}
$$

Family 3 : When, $k=0, \lambda \neq 0, \lambda^{2}-4 k>0$,

$$
\vartheta(\eta)=-\ln \left(\frac{\lambda}{e^{\lambda(\eta+E E)}-1}\right) .
$$

Family 4: When, $k \neq 0, \lambda \neq 0, \lambda^{2}-4 k=0$,

$$
\vartheta(\eta)=\ln \left(-\frac{2 \lambda(\eta+E E)+4}{\lambda^{2}(\eta+E E)}\right) .
$$

Family 5: When, $k=0, \lambda=0, \lambda^{2}-4 k=0$,

$\vartheta(\eta)=\ln (\eta+E E)$,

Where, EE is a integral constant.

Step 3: By substituting Eq. (5) and its derivatives into Eq. (4), we get algebraic equation system. This system was solved by using the Mathematica software program and then the solutions of the MBQ equation were obtained.

\section{Application}

In this section, the modified expansion function method will be used to obtain solutions of the MBQ equation. Consider the following travelling wave transformation: $u(x, t)=u(\xi), \quad \xi=v(x-c t)$

the following nonlinear ordinary differential equation is obtained,

$a u^{2}+2\left(c^{2}-1\right) u-2 c^{2} v^{2} u^{\prime \prime}=0$.

If the balancing procedure is applied to equation (13), we get $n=m+2$ equality.

Choosing $m=1$, we get $n=3$. Eq. (5) for $m$ and $n$ values is obtained as follows;

$u(\xi)=\frac{A_{0}+A_{1} e^{-\vartheta}+A_{2} e^{-2 \vartheta}+A_{3} e^{-3 \vartheta}}{B_{0}+B_{1} e^{-\vartheta}}$

If Eq. (14) is regulated according to the necessary term in equation (13), then the following system of algebraic equations is obtained which consists of the coefficients of $e^{-\vartheta(\xi)}$.

Some suitable coefficients obtained by using the Mathematica package program are given below.

Case-1:

$A_{0}=\frac{12 \mu v^{2} B_{0}}{a-a\left(\lambda^{2}-4 \mu\right) v^{2}}$,

$A_{1}=-\frac{12 v^{2}\left(\lambda B_{0}+\mu B_{1}\right)}{a\left(-1+\left(\lambda^{2}-4 \mu\right) v^{2}\right)}$,

$A_{2}=-\frac{12 v^{2}\left(B_{0}+\lambda B_{1}\right)}{a\left(-1+\left(\lambda^{2}-4 \mu\right) v^{2}\right)}$,

$A_{3}=\frac{12 v^{2} B_{1}}{a-a\left(\lambda^{2}-4 \mu\right) v^{2}}$,

$c=\frac{1}{\sqrt{1-v^{2}\left(\lambda^{2}-4 \mu\right)}}$.

Substituting these coefficients into Eq. (14), the following solutions:

Family 1: When, $k \neq 0, \lambda^{2}-4 k>0$, solution of equation (1),

$u_{1}(x, t)=\frac{\left(12\left(\lambda^{2}-4 \mu\right) \mu v^{2}\right)}{\left(a\left(-1+\left(\lambda^{2}-4 \mu\right) v^{2}\right)\left(\lambda \operatorname{Cosh}[\psi]+\sqrt{\lambda^{2}-4 \mu} \operatorname{Sinh}[\psi]\right)^{2}\right)}$,

where,

$\left(\psi=\left[\frac{1}{2} \sqrt{\lambda^{2}-4 \mu}(E E+\xi) v\right]\right)$. 

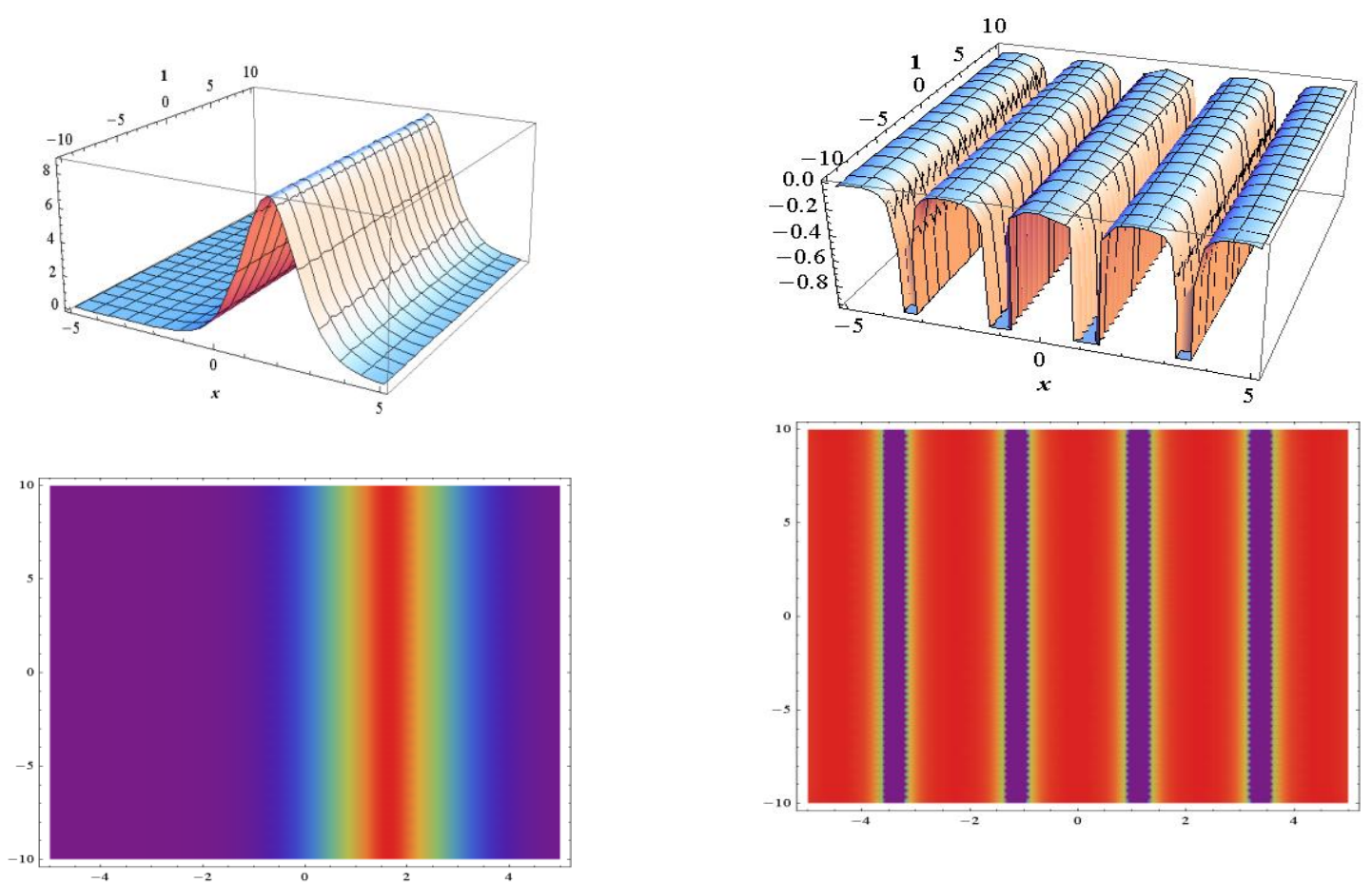

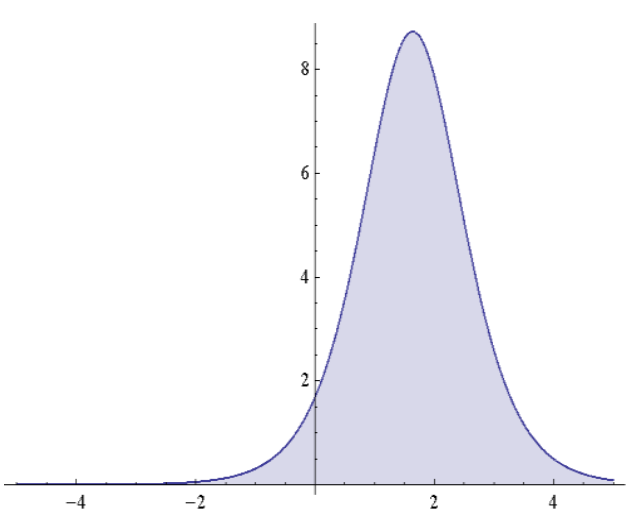

Figure-1. The 3D, density graphic and 2D surfaces of Eq. (15) in $\lambda=2, \mu=0.2, c=4, a=0.5, v=1, E E=0.75$ and $t=1$

Family 2: When, $k \neq 0, \lambda^{2}-4 k<0$, we get,

$u_{2}(x, t)=\frac{\left(12\left(\lambda^{2}-4 \mu\right) \mu v^{2}\right)}{\left(a\left(-1+\left(\lambda^{2}-4 \mu\right) v^{2}\right)\left(\lambda \operatorname{Cos}[v]-\sqrt{\left.\left.-\lambda^{2}+4 \mu \operatorname{Sin}[v]\right)^{2}\right)}\right.\right.}$,

$u_{3}(x, t)=\left(-\frac{3 \lambda^{2} v^{2} \operatorname{Csch}\left[\frac{1}{2} \lambda(E E+(\xi) v)\right]^{2}}{-a+a \lambda^{2} v^{2}}\right)$

Figure-2. The 3D, density graphic and 2D surfaces of Eq. (16) in $\lambda=2, \mu=0.2, c=4, a=0.5, v=1, E E=0.75$ and $t=1$.

Family 3: $k=0, \lambda \neq 0, \lambda^{2}-4 k>0$,

where,

$\left(v=\left[\frac{1}{2} \sqrt{-\lambda^{2}+4 \mu}(E E+\xi) v\right]\right)$. 

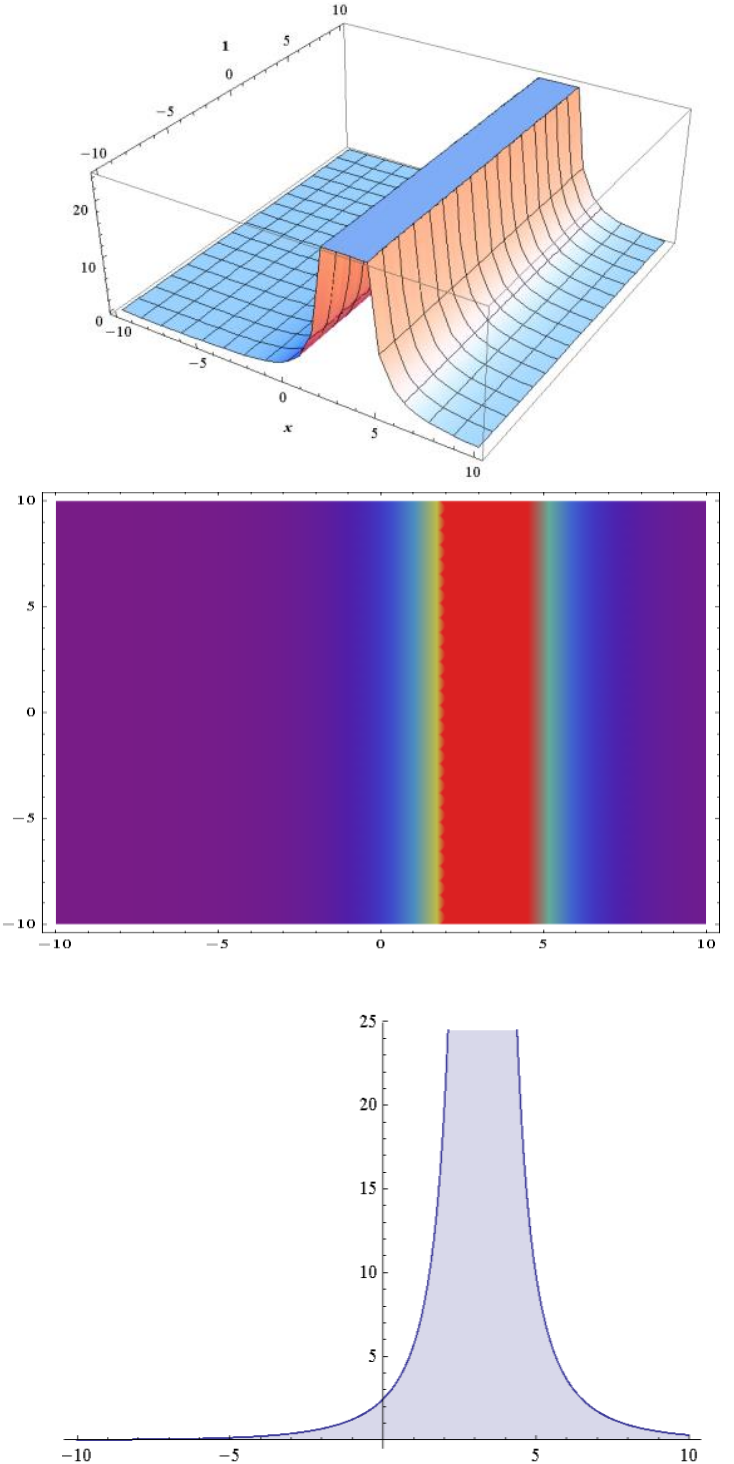

Figure-3. The 3D, density graphic and 2D surfaces of Eq. (17) in $\lambda=0.5, \mu=0, c=4, a=0.5, v=1, E E=0.75$ and $t=1$.

According to Family-4, the solution does not exist.

Family 5: When $k=0, \lambda=0$ and $\lambda^{2}-4 k=0$,

$$
u_{5}(x, t)=\left(\frac{12 v^{2}}{a(E E+(\xi) v)^{2}}\right) \text {, }
$$

\section{Case-2:}

$A_{0}=\frac{\left(\left(-1+c^{2}\right)\left(-\sqrt{c^{4}\left(\lambda^{2}-4 \mu\right)^{2}}+c^{2}\left(\lambda^{2}+8 \mu\right)\right) B_{0}\right)}{a \sqrt{c^{4}\left(\lambda^{2}-4 \mu\right)^{2}}}$,
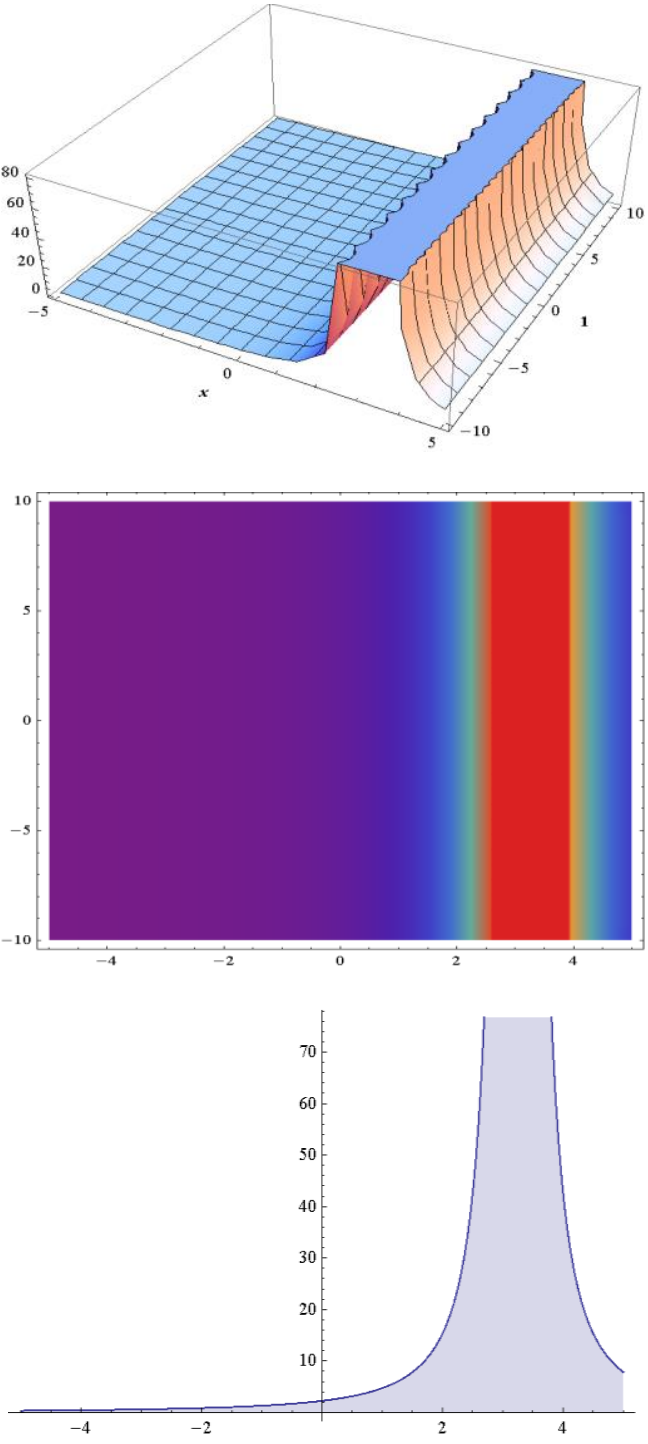

Figure-4. The 3D, density graphic and 2D surfaces of Eq (18) in $\lambda=0, \mu=0, c=4 a=0.5, v=1, E E=0.75$ and $t=1$.

$A_{1}=\frac{\left(\left(-1+c^{2}\right)\left(12 c^{2} \lambda B_{0}+\left(-\sqrt{c^{4}\left(\lambda^{2}-4 \mu\right)^{2}}+c^{2}\left(\lambda^{2}+8 \mu\right)\right) B_{1}\right)\right)}{a \sqrt{c^{4}\left(\lambda^{2}-4 \mu\right)^{2}}}$,

$A_{2}=\frac{\left(12 c^{2}\left(-1+c^{2}\right)\left(B_{0}+\lambda B_{1}\right)\right)}{a \sqrt{c^{4}\left(\lambda^{2}-4 \mu\right)^{2}}}$,

$A_{3}=\frac{\left(12 c^{2}\left(-1+c^{2}\right) B_{1}\right)}{a \sqrt{c^{4}\left(\lambda^{2}-4 \mu\right)^{2}}}$,

$v=-\frac{\sqrt{-1+c^{2}}}{\left(\sqrt{c^{4}\left(\lambda^{2}-4 \mu\right)^{2}}\right)^{\frac{1}{4}}}$. 
Substituting these coefficients into Eq. (14), the following solutions:

Family 1: When, $k \neq 0, \lambda^{2}-4 k>0$, we get

$u_{1}(x, t)=\frac{\left(-1+c^{2}\right) \operatorname{Sech}[\psi]^{2}\left(-2\left(5 c^{2}(\chi)+\sqrt{c^{4}(\chi)^{2}}\right) \mu\right)}{\left(a \sqrt{c^{4}(\chi)^{2}}(\lambda+\sqrt{\chi} \operatorname{Tanh}[\psi])^{2}\right)}$,

(19)

where,

$$
\left(\chi=\lambda^{2}-4 \mu, \psi=\sqrt{\lambda^{2}-4 \mu} \operatorname{Tanh}\left[\frac{1}{2} \sqrt{\lambda^{2}-4 \mu}(E E+\xi)\right]\right) \text {, }
$$
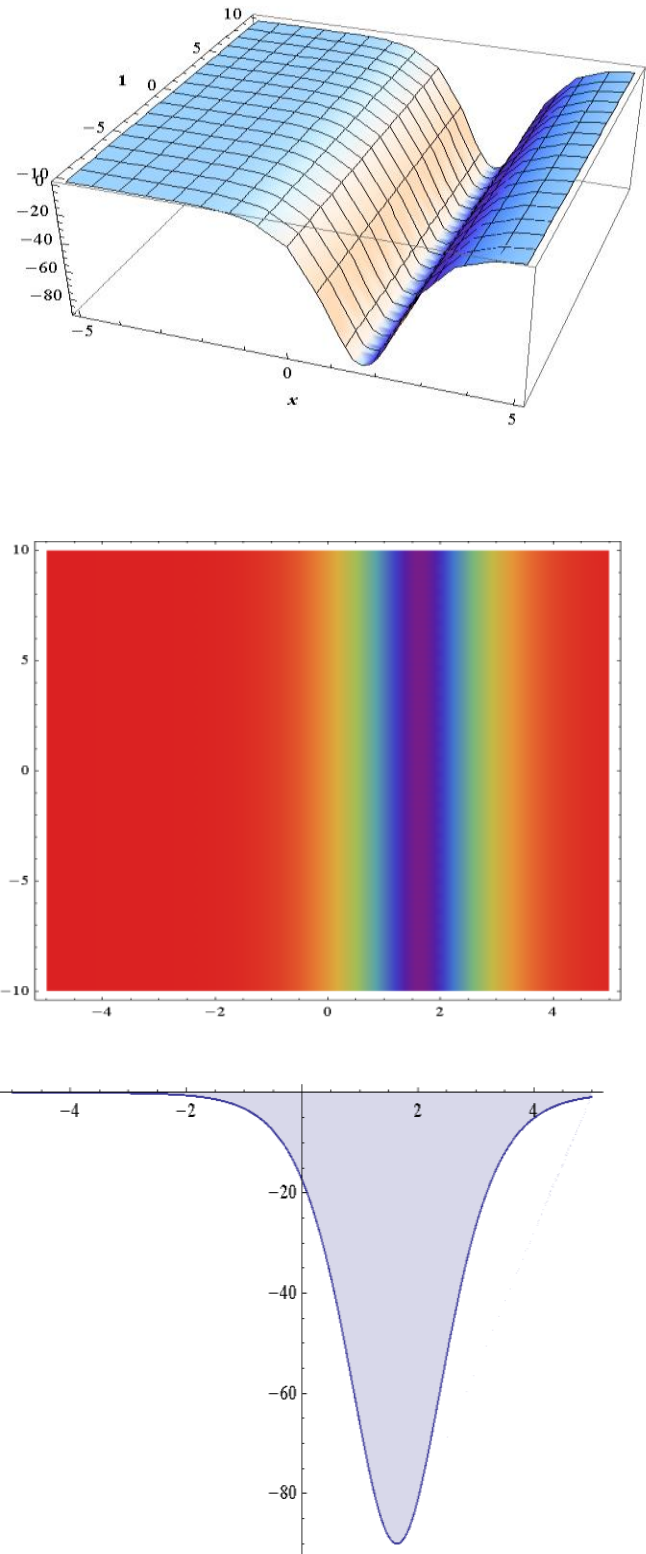

Figure-5. The 3D, density graphic and 2D surfaces of Eq. (19) in $\lambda=0.2, \mu=2, c=4 a=0.5, v=1, E E=0.75$ and $t=1$.
Family 2: When, $k \neq 0, \lambda^{2}-4 k<0$,

$$
\begin{array}{r}
-\left(-1+c^{2}\right)\left(\sqrt{c^{4} \chi^{2}}(\lambda-\sqrt{-\chi} \operatorname{Tan}[\tau])^{2}\right)+ \\
u_{2}(x, t)=\frac{+c^{2} \chi\left(-\lambda^{2}+12 \mu+2 \lambda \sqrt{-\chi} \operatorname{Tan}[\tau]\right)}{\left(\left(\lambda^{2}+8 \mu\right) \operatorname{Tan}[\tau]^{2}\right)},
\end{array}
$$

where,
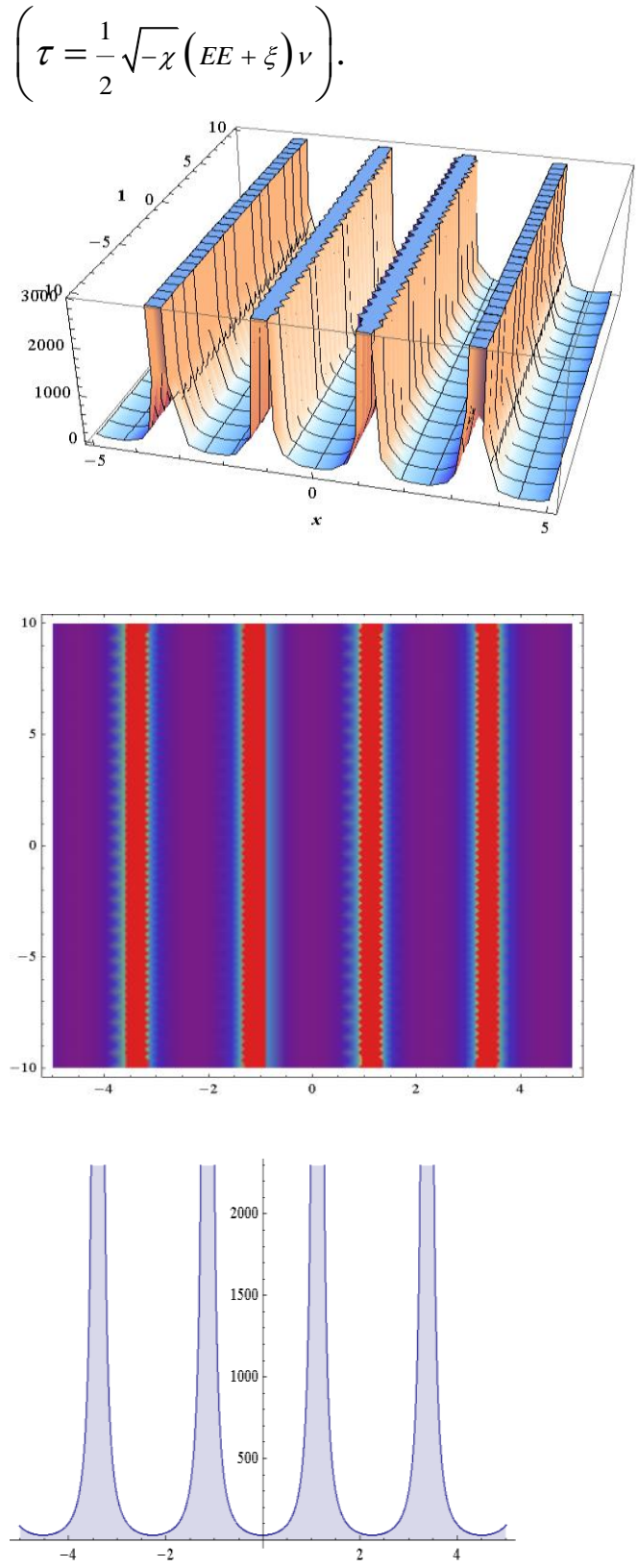

Figure-6. The 3D, density graphic and 2D surfaces of Eq. (20) in $\lambda=0.5, \mu=2, c=4 a=0.5, v=1, E E=0.75$ and $t=1$. 
Family 3: $k=0, \lambda \neq 0, \lambda^{2}-4 k>0$,

$u_{3}(x, t)=\frac{\left(-1+c^{2}\right)\left(-\sqrt{c^{4} \lambda^{4}}+c^{2} \lambda^{4}\left(1+3 \operatorname{Csch}\left[\frac{1}{2} \lambda(E E+\xi) v\right]^{2}\right)\right)}{a \sqrt{c^{4} \lambda^{4}}}$,
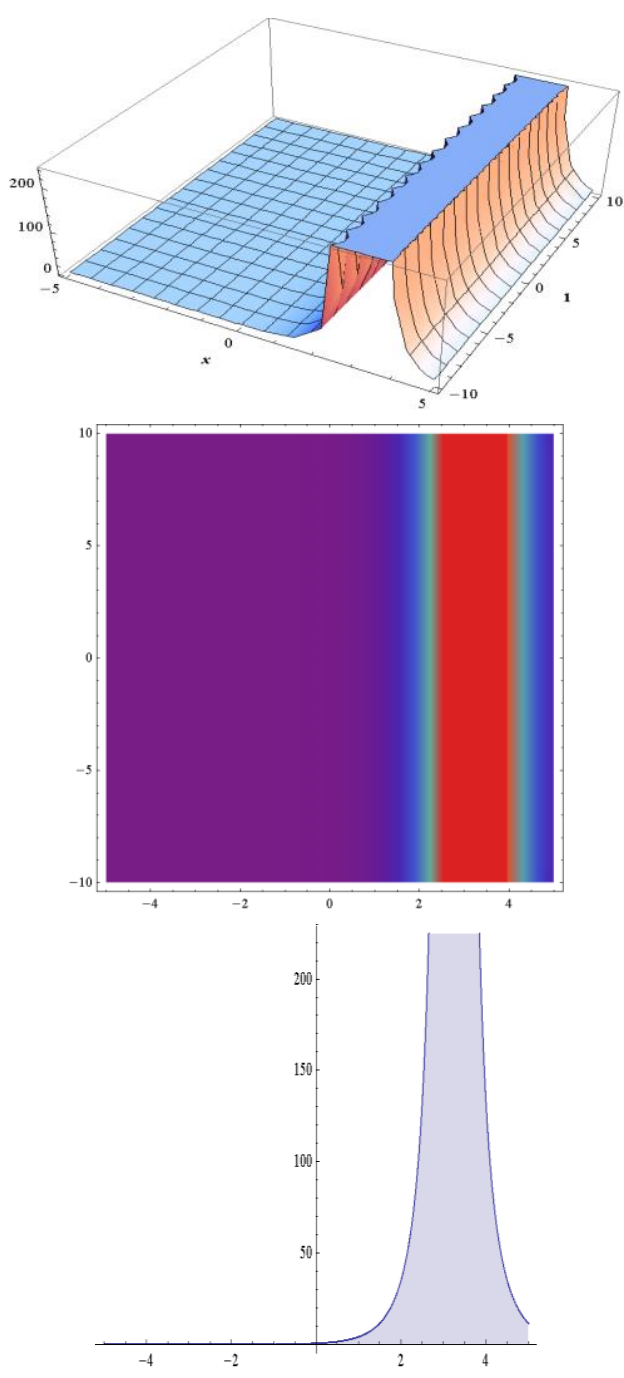

Figure-7. The 3D, density graphic and $2 \mathrm{D}$ surfaces of Eq. (21) in $\lambda=0, \mu=2, c=4 a=0.5, v=1, E E=0.75$ and $t=1$. Family-4 and Family-5, the solution does not exist.

\section{Conclusion}

In this study, we obtained some travelling wave solutions of Boussinesq equation by using modified expansion function method. The results show that the modified expansion function method is a suitable mathematical method for solving nonlinear partial differential equations. The resulting solutions were checked with the Mathematica software. These solutions have been obtained by MEFM for the first time in the literature.

\section{Acknowledgments}

We would like to thank the reviewers and editors who participated in the evaluation process of our production.

\section{References}

[1] Liu, C. S. (2005). Trial equation method and its applications to nonlinear evolution equations, Acta Physica Sinica, 54(6), 2505-2509.

[2] Shen, G., Sun, Y., \& Xiong, Y. (2013). New travelling-wave solutions for Dodd-Bullough equation, Journal of Applied Mathematics, vol. 2013, Article ID 364718, 5 pages.

[3] Sun, Y. (2014). New travelling wave solutions for Sine-Gordon equation, Journal of Applied Mathematics, vol. 2014, Article ID 841416, 4 pages.

[4] Bulut, H., Akturk, T., \& Gurefe, Y. (2015). Travelling wave solutions of the $(\mathrm{N}+1)$-dimensional sine-cosine-Gordon equation, AIP Conference Proceedings 1637, 145.

[5] Akturk, T., Bulut, H., \& Gurefe, Y. (2017). New function method to the $(\mathrm{n}+1)$-dimensional nonlinear problems, An International Journal of Optimization and Control: Theories \& Applications, 7(3), 234239.

[6] Akturk, T., Bulut, H., \& Gurefe, Y. (2017). An application of the new function method to the Zhiber-Shabat equation, An International Journal of Optimization and Control: Theories \& Applications, 7(3), 271-274.

[7] Pandir, Y., Gurefe, Y., Kadak, U., \& Misirli, E. (2012). Classification of exact solutions for some nonlinear partial differential equations with generalized evolution, Abstract and Applied Analysis, vol. 2012, Article ID 478531, 16 pages.

[8] Kudryashov, N. A. (2012). One method for finding exact solutions of nonlinear differential equations, Communications in Nonlinear Science and Numerical Simulation, 17(6), 2248-2253.

[9] Chen, Y., \& Yan, Z. (2005). New exact solutions of $(2+1)$-dimensional Gardner equation via the new sine-Gordon equation expansion method. Chaos, Solitons \& Fractals, 26(2), 399-406.

[10] Baskonus, H. M., Bulut, H., \& Sulaiman, T. A. (2017). Investigation of various travelling wave solutions to the extended (2+1)-dimensional quantum ZK equation. The European Physical Journal Plus, 132(11), 482.

[11] Baskonus, H. M., Bulut, H., \& Atangana, A. (2016). On the complex and hyperbolic structures of the longitudinal wave equation in a magneto-electroelastic circular rod. Smart Materials and Structures, 25(3), 035022.

[12] He, J. H., \& Wu, X. H. (2006). Exp-function method for nonlinear wave equations. Chaos, Solitons \& Fractals, 30(3), 700-708. 
[13] Xu, F. (2008). Application of Exp-function method to symmetric regularized long wave (SRLW) equation. Physics Letters A, 372(3), 252-257.

[14] Clarkson, P.A. (1986). The Painleve property, a modified Boussinesq equation and a modified Kadomtsev-Petviashvili equation. Physica D: Nonlinear Phenomena, 19(3), 447-450.

[15] Li, S., Zhang, W., \& Bu, X. (2017). Periodic wave solutions and solitary wave solutions of generalized modified Boussinesq equation and evolution relationship between both solutions. Journal of Mathematical Analysis and Applications, 449(1), 96-126.

[16] Levine, H.A., Sleeman, B.D. (1985). A note on the nonexistence of global solutions of initial boundary value problems for the Boussinesq equation, Journal of Mathematical Analysis and Applications, 107, 206-210.

[17] Naher, H., \& Abdullah, F. A. (2013). New approach of $\left(\mathrm{G}^{\prime} / \mathrm{G}\right)$-expansion method and new approach of generalized $\left(\mathrm{G}^{\prime} / \mathrm{G}\right)$-expansion method for nonlinear evolution equation. American Institute of Physics Advances, 3(3), 032116.

T. Akturk is an assistant professor in Department of Mathematics and Science Education, at Ordu University; Ordu(Turkey He obtained his M.Sc. degree from Firat University and Ph.D. degree from Firat University. Him areas of interest are numerical solutions of the linear or nonlinear partial differential equations.

An International Journal of Optimization and Control: Theories \& Applications (http://ijocta.balikesir.edu.tr)

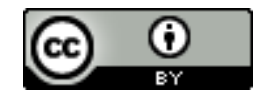

This work is licensed under a Creative Commons Attribution 4.0 International License. The authors retain ownership of the copyright for their article, but they allow anyone to download, reuse, reprint, modify, distribute, and/or copy articles in IJOCTA, so long as the original authors and source are credited. To see the complete license contents, please visit http://creativecommons.org/licenses/by/4.0/. 